\title{
The Role of Ethnicity in Inflammatory Bowel Disease
}

\author{
Gontar Alamsyah Siregar ${ }^{1 *}(\mathbb{D})$, Darmadi Darmadi ${ }^{1}$, Riska Habriel Ruslie $^{2}$ \\ ${ }^{1}$ Department of Internal Medicine, Faculty of Medicine, Universitas Sumatera Utara, Medan, Indonesia; ${ }^{2}$ Department of Child \\ Health, Faculty of Medicine, Universitas Prima Indonesia, Medan, Indonesia
}

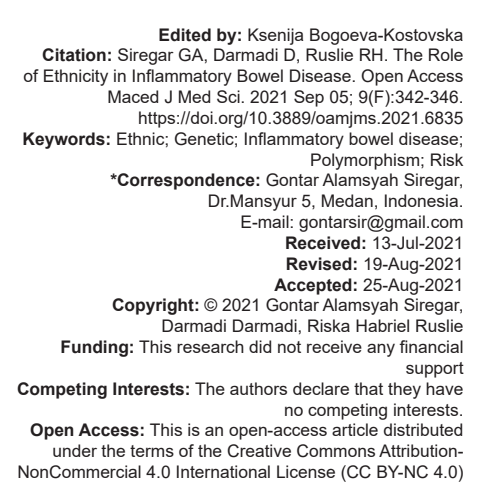

\begin{abstract}
Inflammatory bowel disease (IBD) is a chronic inflammation of the digestive tract which consists of ulcerative colitis and Crohn's disease. The disease is previously recognized as a disease of Western countries but later it spreads all over the world across every ethnicity. The disease manifestations vary from intestinal to extra-intestinal manifestations. There are two risk factors related to the incidence of IBD: Internal and environmental factors. The internal factor is related to genetic susceptibility and genetic susceptibility is associated with ethnicity. Subject from Black ethnic has higher risk for suffering from IBD. Caucasians even have the lowest incidence compared to other ethnics. The disease course is also worse in Black and Hispanic ethnics. Asians have milder disease course. Immigrants tend to have higher risk for IBD compared to native subjects. Further investigations showed that ethnicity carries variable genetic characteristics which affect immune activity, intestinal barrier integrity, and autophagy. All the above mentioned will elicit inflammation if being unbalanced. Chronic inflammation particularly in digestive tract leads to IBD. Knowledge regarding the tendency of IBD in several ethnics raises awareness and will initiate earlier preventive measure against IBD.
\end{abstract}

\section{Introduction}

Inflammatory bowel disease (IBD) is a chronic inflammation of the digestive tract particularly intestine [1], [2]. It consists of two separated entities: ulcerative colitis (UC) and Crohn's disease (CD) [3]. The most common symptoms of IBD are abdominal pain, diarrhea, bloody purulent stool, and weight loss [4], [5]. The occurrence of IBD is related to improper host immune response and environmental factors [1], [6]. The disease has been identified since more than a century ago. UC was first introduced by Samuel Wilks in 1858. CD itself was identified later in 1932 by Burrill Crohn, Leon Ginzburg, and Gordon Oppenheimer [6], [7], [8]. Alleviation of IBD symptoms by steroid administration raised a hypothesis regarding the role of inflammation in the pathogenesis of IBD [7]. The phenomena of increased global IBD incidence are observed recently [9]. The highest prevalence is reported in Western countries [3], [5]. The prevalence is increasing in other part of the world due to industrialization and westernization [6], [8]. The disease has significant negative impact toward patients' quality of life. It also carries heavy economic burden for a country [8].

Two major risk factors for IBD are internal and environmental factors. Internal factor involves genetic predisposition while environmental factor consists of high-fat diet, lack of physical activity, air pollution, good socioeconomic status, and history of appendectomy [3], [8], [9], [10]. Imprudent utilization of antibiotic and non-steroidal anti-inflammatories disrupts the balance of normal flora in gut, triggers inflammation, and leads to IBD [5], [8]. Ethnicity is related to genetic variation in human. The absence of IBD case in Pacific island people and lesser cases in Maori and non-Caucasian suggest that ethnicity is one of determinants of the incidence of IBD [10]. Vegetarian diet [8], [10] and breastfeeding during infancy [8], [9] are some protective factors against IBD. However, neither any single risk factor nor any single protective factor can trigger the incidence of IBD. Complex interaction between those factors is required for the disease to be occurred [9], [10].

The diagnosis of IBD is complex and uneasy. History taking including hereditary predisposition has crucial role in diagnosing the disease. Additional modalities such as physical and rectal examinations, laboratory findings, endoscopy, imaging, and histologic examinations are also valuable [4], [5]. In this article, we will discuss about the relationship between ethnicity and IBD. As ethnicity influences genetic and hereditary predisposition, the knowledge regarding its relationship with IBD will illuminate preventive measures for the disease. 


\section{Epidemiology of IBD}

In 1994, the prevalence of $C D$ and $U C$ in Canada was 198.5/100,000 and 169.7/100,000, respectively. The prevalence was suspected to be higher since there was a proportion of population with IBD who did not seek medical attention [11]. In USA, reported incidence of UC and CD is 12.2 and $10.7 / 100,000$ per year [3]. The incidence of $U C$ and $C D$ in Europe is higher, reaching 24.3 and $12.7 / 100,000$ annually [10]. Even though the incidence of IBD in Asia is low, the trend is increasing [3], [6], [8]. This phenomenon is hypothesized due to the shift in socioeconomic status and significant leap in medical technology [8]. A study in Minnesota, USA showed that the incidence of IBD in whites was 21.6/100,000 person per year compared to $13 / 100,000$ person per year in nonwhites. However, the incidence of IBD in non-whites had increase sharply by time. The non-Hispanics had higher incidence rate compared to Hispanics in a ratio of $4: 3$ [12]. The difference is diminished by migration activity. Immigrants from all regions tend to have lower IBD incidence compared to native ones. Furthermore, immigrants' children have similar incidence of IBD compared to non-immigrants' children [12], [13].

The prevalence of IBD was higher in females compared to males [11] particularly among AfricanAmericans [13]. Another literature reported variable results regarding gender predisposition for the risk of IBD. Males had higher risk for $C D$ in Asian ethnicity [3], [14] while females were dominant in Blacks. In Caucasians and Hispanics, the gender tendency was balanced. This condition was strongly affected by the smoking habit [14]. The disease mainly occurs between second and third decade of life but the majority patient come to healthcare facilities at the third and fourth decade [3], [7], [15]. Based on ethnicity, Hispanics and Asians seem to be diagnosed with IBD at an older age than African-Americans and whites [13].

\section{IBD Manifestations}

Abdominal pain is the most common manifestation of IBD. The pain is chronic and colicky. The pain is often localized in the right lower quadrant of abdomen. It is worsening with bowel movement. Diarrhea may be present with watery or bloody in characteristic. Diarrhea in IBD is chronic and intermittent. Patients may suffer from weight loss due to chronic diarrhea malabsorption of nutrients, and anorexia [5]. Fistula and obstruction are late manifestations [3], [5] along with colorectal cancer. IBD increases the risk of colorectal cancer as high as 2.4 times compared to subjects without IBD [3], [4]. Extra-intestinal manifestations may occur in 6\%-47\% cases [1], [3], [10]. Among all extra- intestinal manifestations, joint manifestations are the most common followed by ocular manifestations [3].

Based on ethnicity, there is a higher tendency of the incidence of $C D$ among AfricanAmericans, Hispanics, and Asians compared to white population [13], [16]. African-Americans and Hispanics with $\mathrm{CD}$ commonly have ileocolonic disease distribution while Asians have both colonic or ileal distribution. Disease extent to the upper gastrointestinal tract is rare in all ethnicities. Patients with UC from AfricanAmerican, Hispanic, and Asian ethnicities tend to suffer from pancolitis than left-sided colitis or proctitis [13]. Complications of disease are also more prominent in coloured skin populations. In contrast with UC, there is no difference in disease manifestation between ethnicities [16]. Regarding IBD-related mortality, AfricanAmericans have the highest rate followed by whites and Hispanics [13]. Socio-cultural differences among each ethnicity may contribute to these conditions [16]. Another literature found that the prevalence of $C D$ and UC is roughly similar between African-American and whites. The disease perception is also different between ethnicities. Whites have better perception compared to African-Americans. African-Americans think that IBD hampers all of their aspects of life harder than whites. Different result may result from different sample population [13], [17]. However, the incidence and prevalence of IBD as a single entity are still most prominent in whites compared to other ethnicities [13].

\section{Pathogenesis of IBD}

The pathogenesis of IBD is associated with dysregulated immune response toward external and internal antigens. The response is related to genetic predisposition [3], [4]. [5], [14]. This theory is supported by the fact that subject with present familial history of IBD has higher tendency for suffering from IBD. Genetic predisposition is in relationship with gene polymorphism, a variation which occurs in certain group of population or ethnicity. However, migration and increased global incidence of IBD recently prove that external antigens also play important role [14]. In patients with CD, T helper (Th)1 and Th17 are highly active and the production of pro-inflammatory cytokines is increasing for example interleukin (IL)-17, interferon (IFN)- $\gamma$, and tumor necrosis factor (TNF)- $\alpha$. Meanwhile in UC, the activity of Th2 is increased leading to B cell and natural killer activation [5]. The state of hyperactive adaptive immune system is jeopardized by increased activity of antigen presenting cells (APC) in the intestinal mucosa, including macrophage and dendritic cell. This condition allows antigen translocation from intestinal lumen [5], [7]. The amount of human leukocyte antigen 
(HLA) on APC's surface is also increased in subject with IBD. Increased HLA expression facilitates antigen processing and presentation [7].

Beside activity of immune system, intact intestinal barrier is important in preventing the occurrence of IBD. Intestinal barrier is made up from between enterocytes, goblet cell, neuroendocrine cell, Paneth cell, and M cell. This barrier is the first physical defense against antigens such as microorganisms and toxins. The damage in this barrier will increase intestinal permeability and permit antigen to enter host's circulation [4], [5]. The balance of microorganism in gut also affects T-regulatory cell activity and epithelial function [5], [7]. Microorganism imbalance causes anti-inflammatory cytokines, such as IL-2, IL-10, TGF- $\beta$, and TNF- $\beta$, to be are downregulated [7]. Inflammation occurred will further impair intestinal barrier and lead to chronic inflammation [4]. Molecular mimicry between foreign antigens and self-antigens contributes to the pathogenesis of IBD. As stated before, foreign antigens trigger immune activation, and when their structures have similarity with self-antigens, chronic immune activation will persist [1]. Autophagy, a process of recycling abnormal cytoplasmic component, is found to be impaired in patients with IBD. One of the abnormal components is pathogen. Prolonged presence of pathogen in cytoplasma elicits prolonged inflammation [4], [5], [8], [18].

Inflammation as the basic pathogenesis of IBD is influenced by genetic factors and genetic factor itself is affected by ethnicity. Compared to Europeans, Asians and African-Americans have more NOD2, ATG16L1, and IL23R genes which are associated with the incidence of $C D$ [13], [16]. Polymorphism in genes regulating TNF (TNFSF15 and TNFSD8) and HLA (HLA-DQ1 and HLA-DQB1) expressions also play important role. The above polymorphisms are observed in Asians and their presence is associated with increased risk for IBD. Genetic factor also determine the presence of side effect from IBD management. For example, myelosuppression is associated with TPMT gen in Europeans and NUDT15 gen in Asians. Difference in tobacco usage is found between ethnicities. African-Americans who suffer from CD have higher rates of smoking in comparison with non-AfricanAmericans patients [16]. Furthermore, they have worse disease course due to the habit. Additionally, AfricanAmericans patients have lower compliance for disease follow-up compared to whites. They tend to visit emergency department and be horpitalized more often. This situation is not observed on Asians and Hispanics [13], [16].

\section{Ethnicity and IBD}

\section{Incidence and disease course}

As stated before that the etiology of IBD is multi-factorial, ethnic and racial variations also play important role [11], [19]. At first, IBD was thought to be associated with Ashkenazi Jewish descendants. Lately, the disease has spread globally across ethnics [14], [15], [19]. Unpredictably, subject from Black ethnic group has higher odds for having $C D$, followed by Asian, Hispanic, and lastly Caucasian. For UC, the prevalence is not significantly different among all ethnics. The rate of surgery after 1 and 5 year of CD diagnosis is highest in Black ethnic. In contrast, the rate is lowest in Asian ethnic, marking a milder course of disease in this population. Meanwhile in UC patients, Hispanic ethnic has the highest rate of surgery at 1 and 5 year after the diagnosis. Particularly in Asian ethnic, the higher prevalence of IBD is observed in Middle East compared to East and South Asia. Furthermore, immigrant Asians tend to have higher risk for having IBD compared to native ones [14]. Other source even stated that immigrants from South Asia have higher incidence and prevalence of IBD compared to indigenous Caucasians in their destinations [19]. A study from Scotland reported that relative risk for UC was higher in Indian and Pakistani males compared to other white ethnicities and even all ethnicities combined. The relative risk for $\mathrm{CD}$ was also high in Pakistani males compared to all other ethnicities [20].

Among Americans, African and white Americans tend to suffer from CD while Mexican Americans predominantly have UC. African-Americans who have $C D$ suffer from extra-intestinal symptoms such as arthritis and uveitis more frequently compared to whites. In the other hand, white Americans with UC have higher tendency for developing joint symptoms and osteoporosis compared to Mexican Americans. The rate of disease complication was not significantly different among the three ethnics [15]. Another study, also in American population, showed that AfricanAmericans and Caucasians have higher rate of $C D$ development compared to Indian American. Consistent with previous study, there was no significant difference observed regarding complication of disease [19].

For the outcome after surgical approach, African-Americans seem to have the worst among all ethnicities. This phenomenon may be related to worse disease course before surgery, such as the presence of sepsis. Therefore, post-operative morbidity and mortality are significantly increased even after adjustment for age, gender, duration of operation, smoking status, and obesity [16].

\section{Effect on genetic factors}

Ethnicity is hypothesized to be associated with genetic polymorphisms. Genetic polymorphisms involving gastrointestinal tract function may cause various disorders including IBD [14], [15], [21]. Polymorphism itself is defined as a variation in the DNA sequence that occurs in a population with a frequency of $1 \%$ or higher. Polymorphism causes changes in 
gene expression related to various diseases and conditions [22]. There are many genetic predispositions associated with the incidence of IBD such as NOD2, IBD1-9, TNFSF15, IL23R, ATG16L1, IRGM, PRDM1, and NDP52 [1]. [3], [8], [18], [23]. The most common investigated genetic predispositions are NOD2, IL23R, and ATG16L1 [8], [23]. The genetic predispositions are found in all ethnics with variable expressions [18]. In Caucasians, several alleles associated with irritable bowel syndrome (IBS) such as NOD2, IL-23R, and ATG16L1 are found to be elevated [14], [15], [19]. However, genetic predisposition cannot elicit IBD without epigenetic mechanisms [4].

Immune hyper-reactivity is associated with methylation in PAR2, RUNX3, TRAF6, IL12B, HLADOB, IL16, IGHG1, and THY1 genes. The methylations induce either elevated pro-inflammatory cytokines or decreased anti-inflammatory cytokines which lead to chronic inflammation in gut [3], [4]. The presence of microRNAs (miRNAs) also influences immune function. Over-expression of miR-155 induces Th1 differentiation while loss of miR-155 is associated with Th2 activity. Over-expression of miR-155 also promotes TNF- $\alpha$, IL-6, IL-12, IL-17, and IFN- $\gamma$ production [3], [4]. miR- 21, another microRNA, is a risk factor for IBD. Its overexpression differentiates naïve $\mathrm{T}$ cell into Th2, supports $\mathrm{T}$ cell-mediated immune responses, and IL-23/Th17 axis [4]. The last miRNA which plays role in the immune response of IBD is miR-146a. It regulates $T$ regulatory cell, dendritic cell, and natural killer cell functions. It is also related to NOD2 and toll-like receptors signaling pathway. NOD2 stimulates pro-inflammatory cytokines levels through NF-KB and caspase 3 signaling pathways along with maintains function of innate and adaptive immunity of intestine [4], [5], [8], [18]. On the other hand, miR-200b acts as protective factor for IBD. It downregulates several pro-inflammatory cytokines such as IL-8 and TNF- $\alpha$ [4]. Initial immune response is correlated with HLA function. Variations in HLA gene will influence the corresponding initial immune response [1].

In regard of intact intestinal barrier, methylation in CHD1, LAMB1, HNF4A, and MYO9B affects epithelial tight junction. Over-expression of miR-21 also impairs intestinal epithelial barrier. miR-21 disrupts tight junctions and increases intestinal permeability, thus allowing antigens to move from lumen of gut [4]. NOD2 also maintains intestinal mucosal barrier, therefore decreased expression of this gene may increase the risk of IBD [4], [5], [8], [18]. Autophagy itself is also regulated by several genes including ATG16L1, NOD2, and IRGM. MicroRNAs such as miR-142-3P, miR196 , and miR-106b hamper the activity of autophagy while miR-93 promotes it [3], [4], [5], [8], [18]. The presence of extraintestinal manifestations of IBD is associated with the presence of HLA-B8/DR3, HLAB35, HLA-B44, HLA-B58, and HLA-DRB1*0103 ${ }^{1}$ while investigation regarding the relationship between
IL23R and TNFSF15 with disease outcome is being conducted [18].

Polymorphism affecting serotonin receptors $5-\mathrm{HT}_{2}, 5-\mathrm{HT}_{3}$, and $5-\mathrm{HT}_{4}$ is associated with IBS. Similar finding is reported regarding $\alpha 2 \mathrm{C}$-adrenoceptor. Those gene polymorphisms related to IBS are only observed in African and Asian but not Caucasian ancestry. Other polymorphisms affecting voltage-gated sodium channel NaV1.5 influence gastrointestinal motility thus increase the risk of IBS. The polymorphisms are more prevalent in Caucasians even though they can be observed in Hispanics, African-Americans, Asians, American-Indians, and Pacific Islanders. In Caucasians and Koreans, polymorphism related to cholecystokinin receptor will increase the susceptibility of IBS. Polymorphism in cannabinoid receptor gene also increase the risk of IBS and this alteration is dominantly found in Korean and Chinese population [21]. The levels of perinuclear antineutrophil cytoplasmic antibody ( $p$-ANCA) were reported to be higher in Mexican Americans compared to whites. The p-ANCA itself increases gastrointestinal inflammation particularly in UC [15].

Polymorphism also affects cytokines. IL-6, a pro-inflammatory cytokine, is increased due to $-174 \mathrm{C} / \mathrm{G}$ polymorphism and the polymorphism itself is associated with Iranian and Indian population. Another pro-inflammatory cytokine, IL-8, plays important role in the gastrointestinal inflammation. Elevation of IL-8 level is associated with $+396 \mathrm{G} / \mathrm{G}$ and $+781 \mathrm{C} / \mathrm{T}$ polymorphisms. The polymorphisms are prominent in Mexican population but not Caucasian. TNF- $\alpha$ level is involved in the pathogenesis of IBS and its level is affected by $-308 \mathrm{G} / \mathrm{A}$ polymorphism which is more frequent in the Dutch and British patients. Polymorphisms in TNFSF15, TNFSF8, HLA-DQA1, and DQB1 are also associated with elevated TNF level and the incidence of IBD in Asians. Meanwhile in Africans, polymorphisms in ZNF649 and LSAMP are the most prominent risk for IBD in association with TND expression. Anti-inflammatory cytokine such as IL-10 was decreased in patients with IBS. The decrease is associated with $-1082 \mathrm{~A}$ and $-592 \mathrm{~A} / \mathrm{C}$ polymorphisms in Asians but not Caucasians [21].

\section{Conclusion}

IBD is increasing in global incidence and not exclusive for Caucasians or Western countries anymore. Ethnicity causes genetic variations that affect immune activity, intestinal barrier integrity, and autophagy which contribute to the pathogenesis of IBD. Ethnics with colored skin tend to have higher risk for IBD and suffer from more severe disease course compared to Caucasians. Native ethnic has lower risk for IBD 
compared to immigrants. This proves that external risk factors plays significant role in the pathogenesis of IBD. Healthcare providers should be aware of the probability of IBD in subjects from colored skin ethnicity especially if other risk factors are present. Further study regarding association between ethnicity and external risk factors with the incidence of IBD is mandatory.

\section{References}

1. Greuter T, Vavricka SR. Extraintestinal manifestations in inflammatory bowel disease-epidemiology, genetics and pathogenesis. ExpertRevGastroenterol Hepatol.2019;13:307-17. https://doi.org/10.1080/17474124.2019.1574569 PMid:30791773

2. Ruslie RH, Darmadi D. Administration of neem (Azadirachta indica A. Juss) leaf extract decreases TNF-a and IL-6 expressions in dextran sodium sulfate-induced colitis in rats. J Adv Vet Anim Res. 2020;7(4):744-9. https://doi.org/10.5455/javar.2020.g476 PMid:33409321

3. Mak WY, Zhao M, Ng SC, Burisch J. The epidemiology of inflammatory bowel disease: East meets west. J Gastroenterol Hepatol. 2020;35(3):380-9. https://doi.org/10.1111/jgh.14872 PMid:31596960

4. Zeng Z, Mukherjee A, Zhang $\mathrm{H}$. From genetic to epigenetics, roles of epigenetics in inflammatory bowel disease. Front Genet. 2019;10:1017. https://dx.doi.org/10.3389\%2Ffgene.2019.01017 PMid:31737035

5. Flynn S, Eisenstein S. Inflammatory bowel disease presentation and diagnosis. Surg Clin North Am. 2019;99(6):1051-62. https:// doi.org/10.1016/j.suc.2019.08.001

PMid:31676047

6. Ng SC, Shi HY, Hamidi N, Underwood FE, Tang W, Benchimol El, et al. Worldwide incidence and prevalence of inflammatory bowel disease in the $21^{\text {st }}$ century: A systematic review of population-based studies. Lancet. 2017;390():2769-78. https:// doi.org/10.1016/s0140-6736(17)32448-0

PMid:29050646

7. Malik TA, Inflammatory bowel disease historical perspective, epidemiology, and risk factors. Surg Clin North Am. 2015;95(6):1105-22. https://doi.org/10.1016/j.suc.2015.07.006 PMid:26596917

8. Kaplan GG. The global burden of IBD: From 2015 to 2025. Nat Rev Gastroenterol Hepatol. 2015;12(12):720-7. https://doi. org/10.1038/nrgastro.2015.150

PMid:26323879

9. Gearry RB, Richardson AK, Frampton CM, Dodgshun AJ, Barclay ML. Population-based cases control study of inflammatory bowel disease risk factors. J Gastroenterol Hepatol. 2010;25(2):325-33. https://doi. org/10.1111/j.1440-1746.2009.06140.x

PMid:20074146

10. Amarapurkar AD, Amarapurkar DN, Rathi P, Sawant P, Patel N, Kamani $P$, et al. Risk factors for inflammatory bowel disease: A prospective multi-center study. Indian J Gastroenterol. 2018;37(3):189-95. https://doi.org/10.1007/s12664-018-0850-0 PMid:29987750

11. Bernstein CN, Blanchard JF, Rawsthorne $P$, Wajda $A$.
Epidemiology of Crohn's disease and ulcerative colitis in a central Canadian province: A population-based study. Am J Epidemiol. 1999;149(10):916-24. https://doi.org/10.1093/ oxfordjournals.aje.a009735

PMid: 10342800

12. Aniwan S, Harmsen WS, Tremaine WJ, Loftus EV. Incidence of inflammatory bowel disease by race and ethnicity in a population-based inception cohort from 1970 through 2010. Therap Adv Gastroenterol. 2019;12:1756284819827692. https://doi.org/10.1177/1756284819827692 PMid:30792818

13. Afzali A, Cross RK. Racial and ethnic minorities with inflammatory bowel disease in the United States: A systematic review of disease characteristics and differences. Inflamm Bowel Dis. 2016;22(8):2023-40. https://doi.org/10.1097/ mib.0000000000000835 PMid:27379446

14. ShiH, LevyAN, TrivediHD, Chan FK, NgSC,AnanthakrishnanAN. Ethnicity influences phenotype and outcomes in inflammatory bowel disease: A systematic review and meta-analysis of population-based studies. Clin Gastroenterol Hepatol. 2018;16(2):190-7. https://doi.org/10.1016/j.cgh.2017.05.047 PMid:28603049

15. Basu D, Lopez I, Kulkarni A, Selin JH. Impact of race and ethnicity on inflammatoryboweldisease.AmJGastroenterol.2005;100(10):225461. https://doi.org/10.1111/j.1572-0241.2005.00233.x PMid: 16181378

16. Barnes EL, Loftus EV, Kappelman MD. Effects of race and ethnicity on diagnosis and management of inflammatory bowel disease. Gastroenterology. 2021;160(3):677-89. https://doi. org/10.1053/j.gastro.2020.08.064 PMid:33098884

17. Finlay DG, Basu D, Sellin JH. Effect of race and ethnicity on perceptions of inflammatory bowel disease. Inflamm Bowel Dis. 2006;12(6):503-7. https://doi. org/10.1097/00054725-200606000-00010 PMid: 16775495

18. McGovern $\mathrm{D}$, Kugathasan $\mathrm{S}$, Cho JH. Genetics of inflammatory bowel diseases. Gastroenterology. 2015;149(5):1163-76.e2. https://doi.org/10.1053/j.gastro.2015.08.001 PMid:26255561

19. Mukewar S, Wu X, Lopez R, Kiran RP, Remzi FH, Shen B. Ethnicity and the risk of development of Crohn's disease of the ileal pouch. J Crohns Colitis. 2013;7(5):e178-85. https://doi. org/10.1016/j.crohns.2012.08.002 PMid:22939817

20. Bhopal RS, Cezard G, Bansal N, Ward HJ, Bhala N. Ethnic variations in five lower gastrointestinal diseases: Scottish health and ethnicity linkage study. BMJ Open. 2014;4(10):e006120. PMid:25335961

21. Xiao Q, Fang X, Li X, Fei G. Ethnic differences in genetic polymorphism associated with irritable bowel syndrome. World J Gastroenterol. 2020;26(17):2049-63. https://dx.doi. org/10.3748\%2Fwjg.v26.i17.2049 PMid:32536774

22. Karki R, Pandya D, Elston RC, Ferlini C. Defining "mutation" and "polymorphism'intheeraofpersonalgenomics. BMCMedGenomics. 2015;8:37. https://dx.doi.org/10.1186\%2Fs12920-015-0115-z

23. Huang $H$, Fang $M$, Jostins $L$, Mirkov MU, Boucher $G$, Anderson CA, et al. Fine-mapping inflammatory bowel disease loci to single variant resolution. Nature. 2017;547(7662):173-8. https://doi.org/10.1038/nature22969 PMid:28658209 